\title{
Paralysis and delayed Z-disc formation in the Xenopus tropicalis unc45b mutant dicky ticker
}

Timothy J Geach, Lyle B Zimmerman*

\begin{abstract}
Background: The protein components of mature skeletal muscle have largely been characterized, but the mechanics and sequence of their assembly during normal development remain an active field of study. Chaperone proteins specific to sarcomeric myosins have been shown to be necessary in zebrafish and invertebrates for proper muscle assembly and function.

Results: The Xenopus tropicalis mutation dicky ticker results in disrupted skeletal muscle myofibrillogenesis, paralysis, and lack of heartbeat, and maps to a missense mutation in the muscle-specific chaperone unc $45 b$. Unc $45 b$ is known to be required for folding the head domains of myosin heavy chains, and mutant embryos fail to incorporate muscle myosin into sarcomeres. Mutants also show delayed polymerization of $\alpha$-actinin-rich Z-bodies into the Z-disks that flank the myosin-containing A-band.
\end{abstract}

Conclusions: The dicky ticker phenotype confirms that a requirement for myosin-specific chaperones is conserved in tetrapod sarcomerogenesis, and also suggests a novel role for myosin chaperone function in Z-body maturation.

\section{Background}

The sarcomere is the fundamental unit of the muscle myofibril, and mediates ATP-dependent contraction and relaxation. While the components of sarcomeres are well understood, the mechanics and sequence of their assembly remain under debate. A key early process in myofibrillogenesis is the polymerization of $\alpha$ actinin-rich Z-bodies into the Z-discs that flank the myosin-containing A-band. In a mature vertebrate sarcomere, Z-discs are held in register with the central M-band by titin filaments tightly associated with filamentous actin $[1,2]$.

Molecular chaperones, which mediate specific protein maturation events, have recently been shown to play important roles in vertebrate myofibrillogenesis. Originally identified as an uncoordinated nematode mutation $[3,4]$, the muscle-specific chaperone unc $45 \mathrm{~b}$ cooperates with hsp90 to fold the N-terminal globular motor (S1) domain of sarcomeric myosins [5-7]. Depletion of these gene functions in zebrafish results in absence of integrated myosin in the sarcomere [8-11], but no effects on Z-disc formation were noted. While in vitro studies of

\footnotetext{
* Correspondence: Izimmer@nimr.mrc.ac.uk

Division of Developmental Biology, MRC-National Institute for Medical Research, The Ridgeway, Mill Hill, London, NW7 1AA, UK
}

these chaperones in cultured mammalian myoblasts have supported roles in sarcomere formation $[6,12]$, in vivo requirements for these proteins have not been described in vertebrates other than teleost fish.

Vertebrate myofibrillogenesis is readily studied in Xenopus embryos, where most skeletal muscle arises from blocks of mesodermally derived somites running along the flank that eventually contribute to the easilyimaged transparent tadpole tail. Somites form and differentiate in a strict anterior-to-posterior sequence, permitting observation of different stages of muscle formation in a single embryo. Recently, a number of Xenopus tropicalis mutations displaying a range of developmental phenotypes [13-15] including defective cardiac muscle formation [16] have been described.

In one of these mutations, dicky ticker $\left(\operatorname{dit}^{m h 71}\right)$, embryos are completely paralyzed with no heartbeat. Tail muscle shows decreased birefringence under polarized light, consistent with disruption of sarcomeric structures. While the myofibrillogenesis program initiates and muscle myosin is detectable at early stages, sarcomeric myosin heavy chain (MyHC) staining rapidly decreases. Disorganized sarcomeres bounded by $\alpha$-actinin-rich structures eventually form, but the transition from Z-bodies to Z-discs is delayed. In the first use 
of the rapid gynogenesis-based positional cloning strategy developed by Khokha et al, [17] we show here that the dit phenotype is caused by a missense mutation in the myosin chaperone $u n c 45 b$. These results not only demonstrate a conserved role for this protein in vertebrate myofibrillogenesis, but also suggest a novel function for this chaperone in Z-disc formation, a key early step in sarcomerogenesis.

\section{Results}

\section{A Cys to Arg conversion in the UCS domain of unc45b disrupts sarcomerogenesis in dit}

Dit was first identified in a forward genetic screen for Xenopus tropicalis developmental mutations [13]. Homozygous embryos lack a heartbeat (Additional File 1 , Movie S1) and are completely paralysed even after vigorous stimulation (Additional File 2, Movie S2), developing cardiac edema and dying by $\sim$ stage 45 . When visualised under polarized light, dit somites showed a marked reduction in birefringence compared to wild type, suggesting disruption to the normal paracrystalline sarcomere structure (Additional File 3, Figure S1) consistent with defective muscle formation rather than aberrant innervation or metabolism. Phalloidin staining of stage 42 tail muscle likewise showed severe disruption to myofibrils (Additional File 3, Figure S1).

The rapid gynogenetic mapping strategy described in Khokha et al. [17] was used to place dit onto a chromosome and estimate a locus-centromere distance. Briefly, dit and sibling wild type gynogenetic embryos were generated by 'early coldshock' suppression of polar body formation in haploid embryos derived from heterozygous carrier females. Bulk segregant pools of mutant and wild type gynogenotes were then assayed with centromeric SSLPs from the $10 \mathrm{X}$. tropicalis chromosomes [17]. Linkage was detected to marker 016H09 near the centromere of Chromosome 2/Linkage Group 6, but not to other centromeres (Figure 1A). An estimate of the mutation-centromere distance was obtained from the fraction of phenotypically mutant embryos observed (35\%) in gynogenetic clutches, placing the locus $15 \mathrm{cM}$ from the centromere using the formula assuming complete interference $(\mathrm{cM})=50(1-(2 \times$ fraction mutant gynogenotes) ) [18]. SSLPs from this region of the meiotic map as well as additional bespoke SSLPs and SNPs from identified scaffolds were then used for high resolution mapping on embryos obtained from a conventional cross of polymorphic heterozygous carriers. Analysis of 562 mutant embryos (1124 meioses) placed the mutant locus in a $230 \mathrm{~kb}$ interval on scaffold_72 (scaffold_72:967475-1197607) (Figure 1B) containing 4 gene models: ap 2b1, pex12, aldh3a1, and the sarcomeric myosin chaperone $u n c 45 b$, whose loss-of-function phenotype in zebrafish closely resembles dit [9]. Sequence of $u n c 45 b$ from wild type and dit embryos revealed a thymidine to cytidine transition at bp 2335 in the coding sequence, converting $\mathrm{Cys}^{779}$ to Arg (Figure $1 C)$. Conserved in both vertebrate and invertebrate unc 45b orthologs (Figure 1D), Cys ${ }^{779}$ is in the N-terminal UCS (Unc45 Cro1p She4p) domain important for folding myosin in vitro $[5,6]$. Whole mount in situ hybridization (WISH) of wild type embryos shows $u n c 45 b$ is expressed throughout the somites and heart at stage 28 (Figure 2A \&2B), becoming further localised to the jaw, branchial arches and body wall muscles by stage 40 (Figure $2 \mathrm{C} \& 2 \mathrm{D}$ ). The mutation in a conserved cysteine residue, expression during muscle development, and similarity of loss-of-function phenotypes in zebrafish combine to suggest $u n c 45 b$ may underlie the dit phenotype.

\section{Z-disc formation is delayed in dit}

Myofibrillogenesis in dit was investigated further by confocal microscopy. At stage 28, the earliest at which dit can be identified, wild type and mutant embryos were scored, genotyped, and their sarcomeric structures analysed by immunohistochemistry. The oldest (most anterior) somites of wild type sarcomeres showed MyHC (detected by MHC A4.1025) integrating into evenly spaced A-bands (Figure 3A). Dit embryos at this early stage displayed $\mathrm{MyHC}$ staining in disorderly fibrillar structures at levels comparable to wild type siblings (Figure 3B), in contrast with the negligible $\mathrm{MyHC}$ immunoreactivity observed in zebrafish $u n c 45 b$ and hsp 90 a mutants $[9,10]$. Headless myosin species retain the capacity to form thick filaments [19], and it is known that $u n c 45 b$ functions to prevent myosin aggregation [20]. The MyHC staining seen in dit during early myofibrillogenesis may represent transient aggregates of filament intermediates in which head domains remain unfolded and are unable to integrate into the sarcomere.

We then evaluated Z-disc formation in dit embryos; $Z$-disc defects were not described in the zebrafish chaperone mutants steif and sloth $[9,10]$. In stage $28 X$. tropicalis tadpoles, wild type anterior sarcomeres had formed discrete $\alpha$-actinin-stained Z-disc structures (Figure 3C), while mutants lacked Z-discs but showed punctate staining consistent with $\alpha$-actinin-rich Z-body precursors (Figure 3D). Occasional stretches of regularly spaced staining are seen consistent with the onset of sarcomere formation (arrowhead) similar to the premyofibrils described by Sanger et al. [21]. In more posterior somites, sarcomere formation is at an earlier stage. Figure $3 \mathrm{E} \& 2 \mathrm{~F}$ show the border (arrows) between somites 15 and 16 in tails stained with $\alpha$-actinin. In wild type (Figure 3E), Z-discs are beginning to assemble into sarcomeres in the slightly older somite 15 (arrowhead, left) 

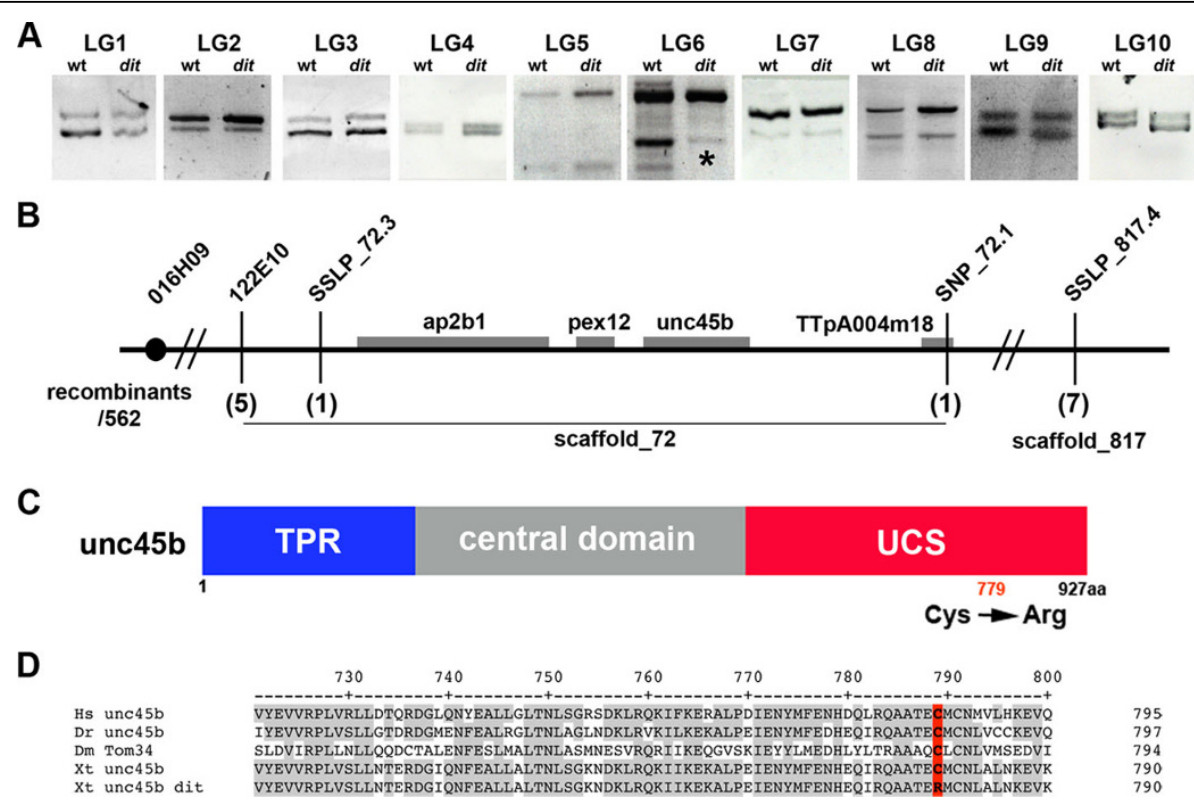

Figure 1 dicky ticker maps to a lesion in the myosin co-chaperone unc45b. (A) Pools of gynogenetically derived wild type and dit embryos were assayed for linkage to polymorphisms near the 10 X. tropicalis chromosomes. Clear linkage $(*)$ was observed at Linkage Group 6 (Chromosome 2) (B) Linkage analysis using SSLP and SNP markers with 562 mutant embryos placed the dit locus in a $230 \mathrm{~kb}$ interval of Scaffold_72 containing four gene models including unc45b. (C) cDNA sequence of dit unc45b revealed a thymidine to cytidine transition, substituting Arg for $\mathrm{Cys}^{779}$ in the UCS domain. (D) Cys ${ }^{779}$ is phylogenetically conserved in unc45b orthologs from Drosophila (Dm) to human $(\mathrm{Hs})$.

while in the adjacent somite 16 no Z-discs are seen. No organized $\alpha$-actinin staining is seen in mutant embryos at these stages in somite 15-16 (Figure 3F).

MyHC is lost and Z-disc formation recovers in dit tadpoles Later in development (stage 43), wild type sarcomeres are organized into paracrystalline structures with $\mathrm{MyHC}$ stained A-bands, $\alpha$-actinin-stained Z-discs, and titinpositive I-bands in register (Figure 4A, C, E). Dit tadpole tails at this stage showed no organized MyHC fibrils (Figure 4B), and the level of immunoreactivity was significantly weaker than wild type, more closely resembling the zebrafish myosin chaperone mutations $[9,10]$. Lower $\mathrm{MyHC}$ protein levels in dit were confirmed by western blots of stage 40 and 43 embryos using another pan-sarcomeric MyHC antibody, F59 (Additional File 4, Figure S2). Sarcomeric MyHC proteins are subject to ubiquitinmediated degradation and autophagy [22-24], and it is likely that the misfolded head domains in dit are actively degraded. Interestingly, Z-discs (marked by both $\alpha$-actinin and titin T12 antibodies) were present in dit tadpole tails at these stages, although these were scattered and severely out of register (Figure 4D \&4F).

\section{Depletion of unc45b phenocopies dit}

To confirm that a deficit in $u n c 45 b$ function underpinned the dit phenotype, we injected two non-overlapping antisense morpholino oligonucleotides (AMO) into wild type embryos. Both a translation blocking (ATG-MO) and a splice blocking AMO deleting $2 / 3$ of the mature protein (Splice-MO) resulted in highly significant numbers of paralysed or partially paralysed tadpoles lacking heartbeat (ATG-MO 42/49 (p < 0.01); Splice-MO 32/34 (P < 0.01), compared to $0 / 52$ in control AMO-injected embryos). Confocal analysis revealed that both the ATG-MO and

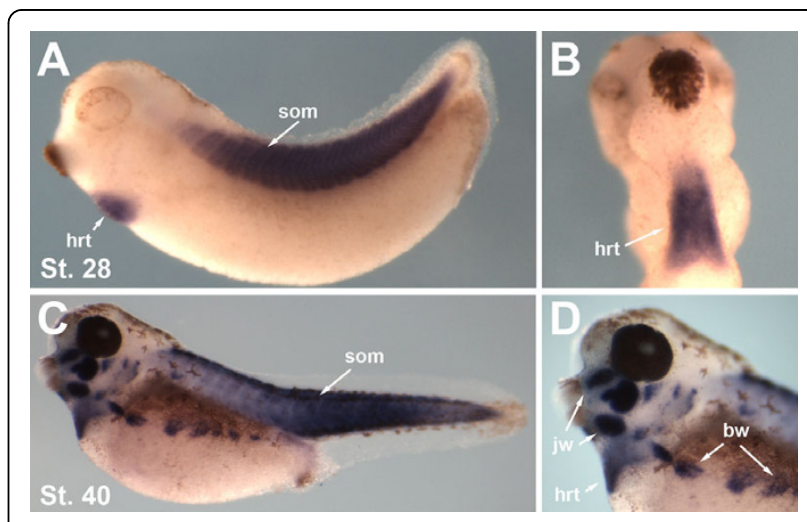

Figure 2 Unc45b expression in Xenopus tropicalis. (A) WISH showing unc45b expression in developing heart (hrt) and somites (som) at stage 28. (B) ventral view of heart expression at stage 28. (C) unc 456 expression at stage 40 throughout the somites and (D) in jaw (jw), heart (hrt) and body wall muscles (bw). 

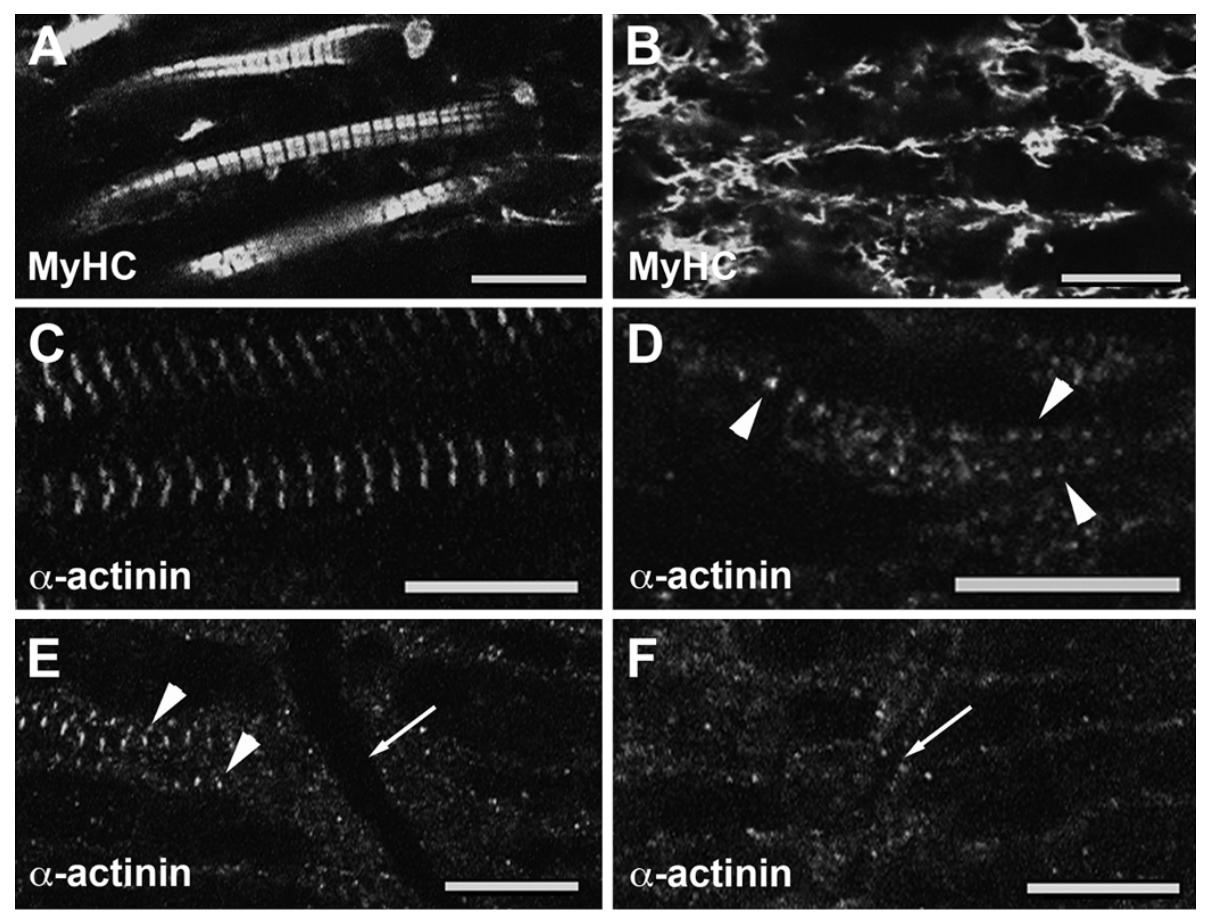

Figure 3 Myofibrillogenesis is disrupted and sarcomere formation is delayed in dit. Confocal analysis of wild type (left panels) and dit embryos (right panels) for sarcomeric myosin heavy chain (MyHC) and Z-disc ( $\alpha$-actinin) immunoreactivity. (A, C) Anterior somites of stage 28 wild type embryos show sarcomeric myosin (MyHC) organized into A-bands separated by regularly spaced Z-discs ( $\alpha$-actinin). (B) Anterior dit somites show disorganized MyHC staining, and (D) $\alpha$-actinin staining is punctate, with occasional ordered Z-bodies consistent with the appearance of incipient sarcomeres (arrowheads). (E) More posteriorly at st. 28, $\alpha$-actinin staining shows wild type somites 15-16 (arrow indicates intersomitic boundary) are beginning to organize Z-discs (arrowheads), while (F) dit somites at this level show only background staining (arrow indicates intersomitic boundary). Scale bars $10 \mu \mathrm{m}$.

Splice-MO produced specific defects in sarcomere formation mirroring those in dit (Figure 5), with reductions in sarcomeric MyHC staining and scattered $\alpha$-actinin-rich $\mathrm{Z}$-disc-like structures. The similarity of the mutant phenotype to those produced by these two independent knockdown experiments is consistent with dit being a strong hypomorph of unc $45 b$.

Interestingly, WISH analysis shows that dit embryos and $u n c 45 b$ antisense-MO injected embryos express increased levels of $u n c 45 b$ mRNA relative to siblings or control MO-injected controls developed in parallel (Figure 6). In zebrafish steif (unc45b) and sloth (hsp90a) mutants, expression of the mutated genes is also upregulated $[9,10]$. The chaperone hsp90 regulates its own expression by holding the transcription factor hsf1 in a latent complex, which is released during heat shock or pharmacological stress when unfolded proteins compete for hsp90 binding [25-27]. The freed hsf1 in turn directly upregulates hsp90 as well as other stress response chaperones [28]. In the dit and steif mutants, persisting unfolded myosin head domains could likewise bind hsp90 and free a heat shock factor to increase chaperone transcription.

\section{Discussion}

While the organization of the mature sarcomere is well understood, the order and mechanism of its assembly remain under debate $[1,29]$. Our data strongly suggest that Z-disc formation, a key early step in myofibrillogenesis, initially requires the presence of mature sarcomeric MyHC proteins. This observation most closely supports the premyofibril model proposed by Du et al. [30] in which closely spaced $\alpha$-actinin-rich Z-bodies are initially separated by non-muscle myosin II; non-muscle myosin II is then replaced by sarcomeric MyHC proteins as these premyofibrils mature, coincident with Z-body alignment and polymerization into Z-discs (reviewed by Sanger et al. [29]). While closely spaced $\alpha$-actininstained Z-bodies are observed in dit sarcomeres, lack of mature sarcomeric MyHC replacement could be responsible for delay of polymerization of these into Z-discs, and Z-disc alignment remains poor. Our data provide less support for the model proposed by Holtzer et al. [31], which hypothesizes that titin 'stitches' together $\alpha$ actinin rich I-Z-I bodies with already formed thick filaments to add sarcomere elements at the ends of Z-disc containing striated myofibrils. This model does not 

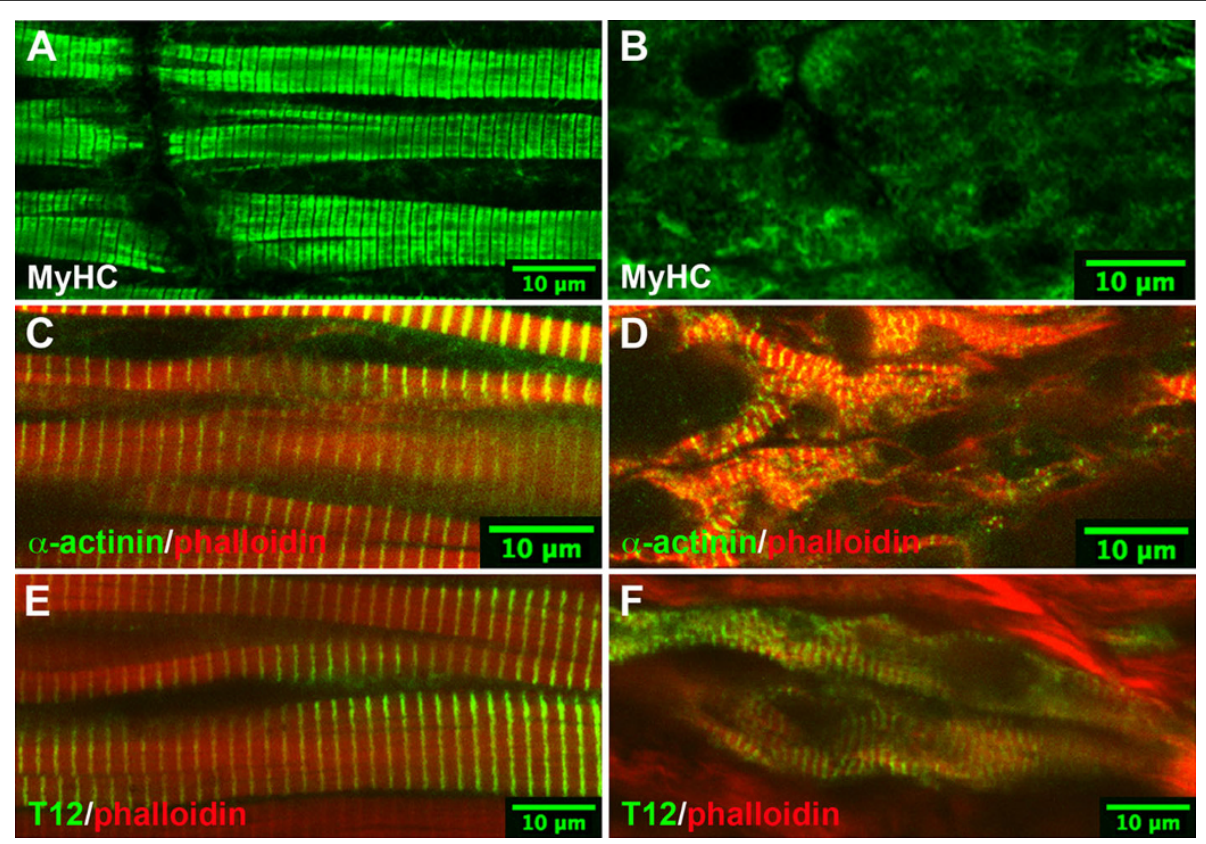

Figure 4 MyHC immunoreactivity is lost and Z-disc formation recovers in dit. Confocal analysis of wild type (left panels) and dit embryos (right panels) stained with MyHC A4.1025, $\alpha$-actinin and titin T12 antibodies. (A, C, E) A-bands and Z-discs are well-organized in wild type somites at stage 43. (B) Sarcomeric myosin staining in dit is virtually absent at these stages, but Z-disc-containing sarcomeres are observed (D, F). Phalloidin stained thin filaments are present but disorganized in dit somites.

anticipate the presence of the regularly spaced premyofibril-like $\alpha$-actinin-stained Z-body structures observed in early dit sarcomeres. The molecular mechanism by which sarcomeric MyHC might participate in Z-disc maturation remains to be determined. While a direct interaction seems unlikely, feedback to Z-bodies via correct $\mathrm{MyHC}$ interdigitation with thin filaments or even contractile activity are also plausible explanations for the requirement for correct $\mathrm{MyHC}$ head domain maturation. Our data also do not rule out the possibility that $u n c 45 b$ functions directly in Z-disc maturation on an unknown protein target; indeed, unc45b protein is known to localize to mature Z-discs in zebrafish [32]. Alternatively, incorrectly folded MyHC heads or
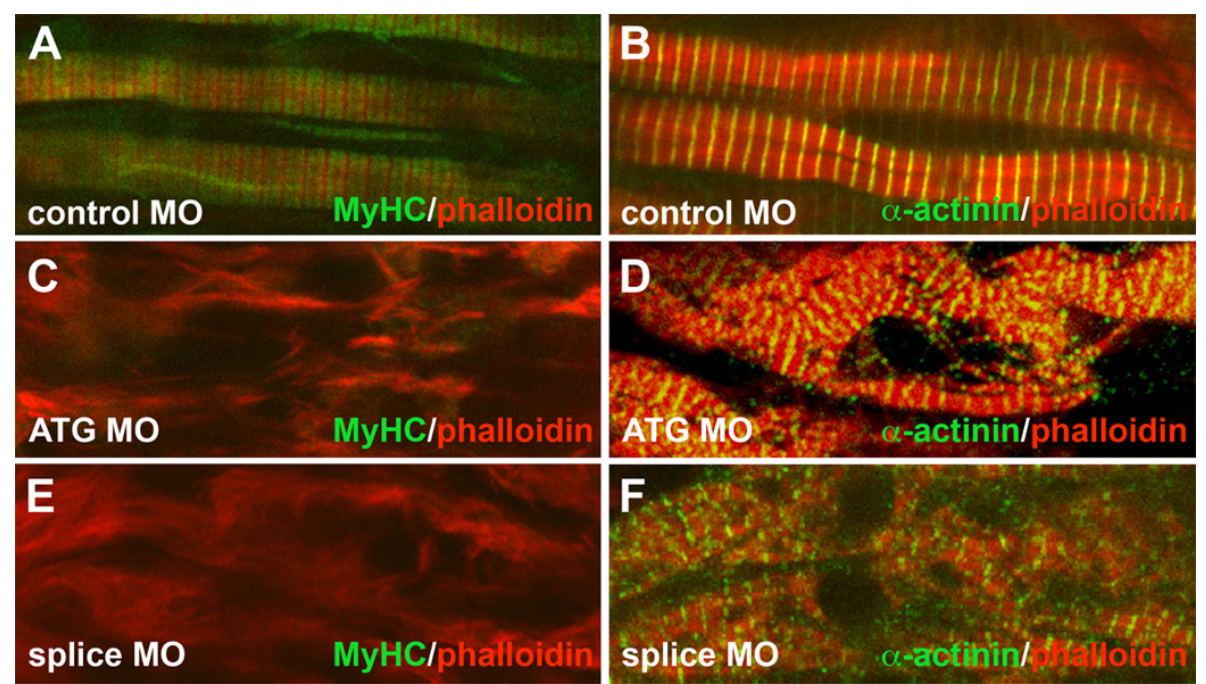

Figure 5 Morpholinos against unc45b phenocopy dit. Unc45b knockdown closely resembles the dit phenotype and results in depletion of MyHC staining and disorganized sarcomeres. Confocal analysis of control MO (A, C), ATG MO-injected (B, D), and splice MO-injected embryos $(\mathbf{E}, \mathbf{F})$ for sarcomeric myosin heavy chain (MyHC) and Z-discs ( $\alpha$-actinin), co-stained with phalloidin. 

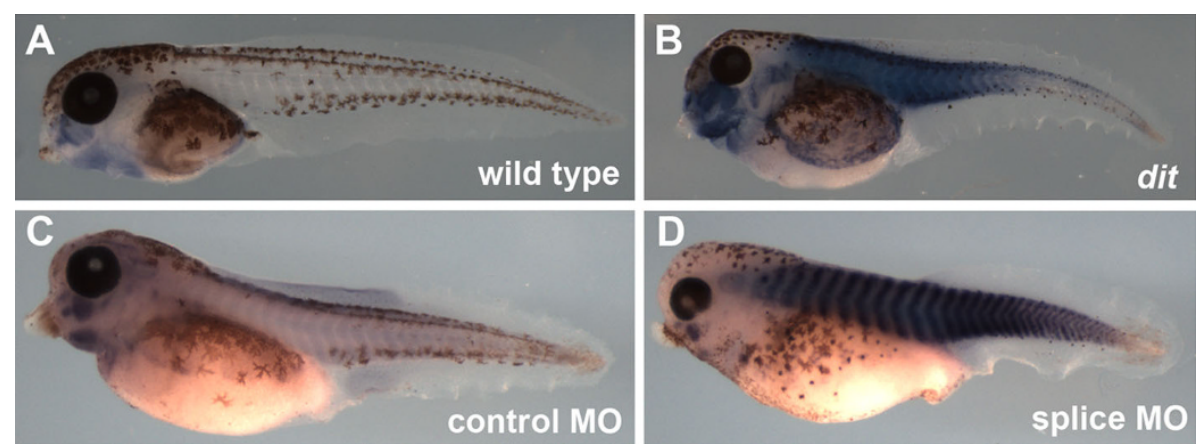

Figure 6 unc45b expression is upregulated in dit. WISH showing expression of unc $45 b$ at stage 43 in (A) wild type and (B) dit, and at stage 40 in (C) control MO and (D) unc45b splice-MO injected embryos. Both splice-MO injected and dit embryos show increased unc45b expression relative to controls.

aggregates could interfere with Z-disc formation in the mutant, with this block released following myosin degradation.

\section{Conclusions}

The $X$. tropicalis dicky ticker mutant demonstrates a conserved role for unc $45 b$ and sarcomeric myosin-specific chaperone function in tetrapod myofibrillogenesis, extending previous phenotypic analysis in teleost fish. A possible novel role for sarcomeric MyHC integration in $\mathrm{Z}$-disc maturation is suggested by the observed delay in aggregation of $\alpha$-actinin staining. Dicky ticker is the second $X$. tropicalis mutation to be cloned, and the first to make use of the rapid gynogenesis-based mapping strategy. Analysis of myofibrillogenesis mutations in X. tropicalis, with its potential for combining genetics with embryological and biochemical tools, promises additional insights into function and regulation of vertebrate muscle assembly.

\section{Methods}

\section{Genetic Mapping of dit}

Following generation of dit embryos by gynogenesis [17] or conventional matings, linkage was analyzed using standard amplification conditions and SSLP markers from the $X$. tropicalis meiotic map http://tropmap.biology.uh.edu[33], together with bespoke SSLPs and SNPs identified in sequence scaffolds of interest. Genomic sequences and scaffolds refer to Joint Genome Institute $X$. tropicalis Assembly v4.1 http://genome.jgi-psf.org/ Xentr4. Centromeric markers were as described by Khokha et al. [17] except LG4-C (001E03), LG8-C (029D12), LG9-C (011H04) and

LG3-C scaffold_163:1658760-1659057),

(F:TTCAGCGAACAGAAGACAACA; R: CAGATG GAGAAATAGTGTTTCACTT;

Bespoke markers for high-resolution mapping were

SSLP_72.3 scaffold_72:967475-967913
F: TTGCCATAAAAGCAGCAGTG; R: TCGCATG CTCAAAATCACTC

SSLP_817.4 scaffold_817:274963-275158

F: TGGCTAGCGGTACCATAAAA; R: ACTTGG TAGGGGTGGCTCTT

SNP_72.1 scaffold_72:1185180-1197607.

F: CATCACGCATAGTCATTATGTTCTT; R: ATT ATGGGATTGCTGGGTGA

A SNP in one allele creates a HaeIII restriction site in the amplified product, which was digested with HaeIII for 2 hours and resolved on a 3\% agarose gel.

Unc $45 b$ cDNA was amplified using Platinum Taq (Invitrogen, UK). Sequencing was performed by Cogenics (UK) and analysed using Lasergene 8. Fulllength wild type cDNA sequence is available at [GenBank: HM053434].

\section{Antisense Morpholino Oligonucleotides}

A translation-blocking AMO (ATG-MO 5'-CCT TTAGCTGCACTGGGTCTTCCAT-3') and a spliceblocking AMO spanning exon-intron boundary 6 (splice-MO 5'-AGCACACATTATTCTTACCCAGTAC$\left.3^{\prime}\right)$ were obtained from GeneTools LLC (Oregon, USA). GeneTools standard control AMO was used for control injections. All were diluted in nuclease free $\mathrm{H}_{2} \mathrm{O}$ and 5 ng injected into both blastomeres at the two-cell stage.

\section{Whole mount in situ hybridization}

WISH was performed according to Harland et al. [34]. A $181 \mathrm{bp}$ antisense probe was made from a $1217 \mathrm{bp}$ fragment of unc $45 b$ (1444 - $2661 \mathrm{bp}$ ) cloned into pCRIITOPO, linearised with HincII and transcribed with Sp6.

\section{Immunohistochemistry}

Embryos were staged according to Nieuwkoop \& Faber (1967), then fixed in 4\% paraformaldehyde. Antibodies used were as follows: MyHC (MHC) A4.1025 [35] and Titin T12 (kind gifts from Elisabeth Ehler (KCL), $\alpha$ - 
actinin (EA-53, Abcam UK), and Alexa ${ }^{\circ}$ fluorophore conjugated secondary antibodies (Invitrogen, UK). Factin was stained with Alexa 543 phalloidin (Invitrogen, UK). Western blots were performed with anti-MyHC F59 [36], obtained from Developmental Studies Hybridoma Bank, University of Iowa, Iowa City, USA, and $\beta$ actin (Sigma, UK) using an HRP conjugated secondary IgG (Santa Cruz, USA). St 28 embryos were dehydrated in methanol and cleared in 2:1 benzyl alcohol:benzyl benzoate for visualization.

\section{Additional material}

Additional file 1: dit embryos lack heartbeat. Beating heart at stage 40 in wild type (top) is not seen in dit (bottom).

Additional file 2: dit tadpoles are paralyzed. Wild type tadpoles at stage 40 are motile (top); dit tadpoles (bottom) are paralyzed.

Additional file 3: Muscle structure is disrupted in dit tails. (A) Birefringence of polarized light in stage 43 wild type tail. (B) Birefringence is greatly reduced in dit tails of the same stage. (C) Phalloidin staining of stage 43 wild type tail muscle showing orderly myofibril structure. (D) Phalloidin staining in dit embryo tails shows disorganized myofibrils.

Additional file 4: Western blot analysis of wild type and dit embryos with MyHC F59 antibody. Presence of MyHC as detected by the F59 antibody is reduced in dit compared to wild type embryos at both stages 40 and 43 .

\begin{abstract}
Abbreviations
AMO: antisense morpholino oligonucleotide; Arg: Arginine; Cys: Cysteine; MyHC: myosin heavy chain; SNP: Single Nucleotide Polymorphism; SSLP: Simple Sequence Length Polymorphism; WISH: whole mount in situ hybridization.
\end{abstract}

\section{Acknowledgements}

The authors thank Tim Mohun (NIMR) and Elisabeth Ehler (KCL, London) for antibodies and useful discussion. Additional thanks go to members of the Zimmerman lab for helpful comments. This work was funded by the Medical Research Council, UK (U117560482).

\section{Authors' contributions}

TG \& LZ conceived the project, designed the experiments and wrote the paper. TG performed the experiments. Both authors read and approved the final manuscript.

Received: 9 April 2010 Accepted: 16 July 2010 Published: 16 July 2010

\section{References}

1. Ehler $\mathrm{E}$, Gautel M: The sarcomere and sarcomerogenesis. Adv Exp Med Biol 2008, 642:1-14.

2. Sparrow JC, Schock F: The initial steps of myofibril assembly: integrins pave the way. Nat Rev Mol Cell Biol 2009, 10:293-8.

3. Venolia L, Ao W, Kim S, Kim C, Pilgrim D: unc-45 gene of Caenorhabditis elegans encodes a muscle-specific tetratricopeptide repeat-containing protein. Cell Motil Cytoskeleton 1999, 42:163-77.

4. Venolia L, Waterston RH: The unc-45 gene of Caenorhabditis elegans is an essential muscle-affecting gene with maternal expression. Genetics 1990, 126:345-53.

5. Liu L, Srikakulam R, Winkelmann DA: Unc45 activates Hsp90-dependent folding of the myosin motor domain. J Biol Chem 2008, 283:13185-93.

6. Srikakulam R, Liu L, Winkelmann DA: Unc45b forms a cytosolic complex with Hsp90 and targets the unfolded myosin motor domain. PLoS One 2008, 3:e2137.
7. Srikakulam R, Winkelmann DA: Chaperone-mediated folding and assembly of myosin in striated muscle. J Cell Sci 2004, 117:641-52.

8. Du SJ, Li H, Bian Y, Zhong Y: Heat-shock protein 90alpha1 is required for organized myofibril assembly in skeletal muscles of zebrafish embryos. Proc Natl Acad Sci USA 2008, 105:554-9.

9. Etard C, Behra M, Fischer N, Hutcheson D, Geisler R, Strahle U: The UCS factor Steif/Unc-45b interacts with the heat shock protein Hsp90a during myofibrillogenesis. Dev Biol 2007, 308:133-43.

10. Hawkins TA, Haramis AP, Etard C, Prodromou C, Vaughan CK, Ashworth R, Ray S, Behra M, Holder N, Talbot WS, et al: The ATPase-dependent chaperoning activity of Hsp90a regulates thick filament formation and integration during skeletal muscle myofibrillogenesis. Development 2008, 135:1147-56.

11. Wohlgemuth SL, Crawford BD, Pilgrim DB: The myosin co-chaperone UNC45 is required for skeletal and cardiac muscle function in zebrafish. Dev Biol 2007, 303:483-92.

12. Price MG, Landsverk ML, Barral JM, Epstein HF: Two mammalian UNC-45 isoforms are related to distinct cytoskeletal and muscle-specific functions. J Cell Sci 2002, 115:4013-23.

13. Goda T, Abu-Daya A, Carruthers S, Clark MD, Stemple DL, Zimmerman LB: Genetic screens for mutations affecting development of Xenopus tropicalis. PLoS Genet 2006, 2:e91.

14. Grammer TC, Khokha MK, Lane MA, Lam K, Harland RM: Identification of mutants in inbred Xenopus tropicalis. Mech Dev 2005, 122:263-72.

15. Noramly S, Zimmerman L, Cox A, Aloise R, Fisher M, Grainger RM: A gynogenetic screen to isolate naturally occurring recessive mutations in Xenopus tropicalis. Mech Dev 2005, 122:273-87.

16. Abu-Daya A, Sater AK, Wells DE, Mohun TJ, Zimmerman LB: Absence of heartbeat in the Xenopus tropicalis mutation muzak is caused by a nonsense mutation in cardiac myosin myh6. Dev Biol 2009, 336:20-9.

17. Khokha MK, Krylov V, Reilly MJ, Gall JG, Bhattacharya D, Cheung CY, Kaufman S, Lam DK, Macha J, Ngo C, et al: Rapid gynogenetic mapping of Xenopus tropicalis mutations to chromosomes. Dev Dyn 2009, 238:1398-46.

18. Johnson SL, Africa D, Horne S, Postlethwait JH: Half-tetrad analysis in zebrafish: mapping the ros mutation and the centromere of linkage group I. Genetics 1995, 139:1727-35.

19. Cripps RM, Suggs JA, Bernstein SI: Assembly of thick filaments and myofibrils occurs in the absence of the myosin head. EMBO J 1999, 18:1793-804

20. Barral JM, Hutagalung AH, Brinker A, Hartl FU, Epstein HF: Role of the myosin assembly protein UNC-45 as a molecular chaperone for myosin. Science 2002, 295:669-71.

21. Sanger JW, Wang J, Holloway B, Du A, Sanger JM: Myofibrillogenesis in skeletal muscle cells in zebrafish. Cell Motil Cytoskeleton 2009, 66:556-66.

22. Galvez AS, Diwan A, Odley AM, Hahn HS, Osinska H, Melendez JG, Robbins J, Lynch RA, Marreez Y, Dorn GW: Cardiomyocyte degeneration with calpain deficiency reveals a critical role in protein homeostasis. Circ Res 2007, 100:1071-8.

23. Tannous P, Zhu H, Nemchenko A, Berry JM, Johnstone JL, Shelton JM, Miller FJ, Rothermel BA Jr, Hill JA: Intracellular protein aggregation is a proximal trigger of cardiomyocyte autophagy. Circulation 2008, 117:3070-8.

24. Witt SH, Granzier H, Witt CC, Labeit S: MURF-1 and MURF-2 target a specific subset of myofibrillar proteins redundantly: towards understanding MURF-dependent muscle ubiquitination. J Mol Biol 2005, 350:713-22.

25. Ali A, Bharadwaj S, O'Carroll R, Ovsenek N: HSP90 interacts with and regulates the activity of heat shock factor 1 in Xenopus oocytes. Mol Cell Biol 1998, 18:4949-60.

26. Bharadwaj S, Ali A, Ovsenek N: Multiple components of the HSP90 chaperone complex function in regulation of heat shock factor 1 In vivo. Mol Cell Biol 1999, 19:8033-41.

27. Zou J, Guo Y, Guettouche T, Smith DF, Voellmy R: Repression of heat shock transcription factor HSF1 activation by HSP90 (HSP90 complex) that forms a stress-sensitive complex with HSF1. Cell 1998, 94:471-80.

28. Metchat A, Akerfelt M, Bierkamp C, Delsinne V, Sistonen L, Alexandre H, Christians ES: Mammalian heat shock factor 1 is essential for oocyte meiosis and directly regulates Hsp90alpha expression. I Biol Chem 2009, 284:9521-8. 
29. Sanger JW, Kang S, Siebrands CC, Freeman N, Du A, Wang J, Stout AL, Sanger JM: How to build a myofibril. J Muscle Res Cell Motil 2005, 26:343-54.

30. Du A, Sanger JM, Linask KK, Sanger JW: Myofibrillogenesis in the first cardiomyocytes formed from isolated quail precardiac mesoderm. Dev Biol 2003, 257:382-94.

31. Holtzer H, Hijikata T, Lin ZX, Zhang ZQ, Holtzer S, Protasi F, FranziniArmstrong C, Sweeney HL: Independent assembly of 1.6 microns long bipolar MHC filaments and I-Z-I bodies. Cell Struct Funct 1997, 22:83-93.

32. Etard C, Roostalu U, Strahle U: Shuttling of the chaperones Unc 45b and Hsp90a between the A band and the Z line of the myofibril. J Cell Biol 2008, 180:1163-75.

33. Wells D, Gutierrez L, Xu Z, Krylov V, Macha J, Blankenburg K, Hitchens M, Bellot L, Spivey M, Kowis A, et al: A Genetic Map of Xenopus tropicalis. Developmental Biology .

34. Harland RM: In situ hybridization: an improved whole-mount method for Xenopus embryos. Methods Cell Biol 1991, 36:685-95.

35. Dan-Goor M, Silberstein L, Kessel M, Muhlrad A: Localization of epitopes and functional effects of two novel monoclonal antibodies against skeletal muscle myosin. J Muscle Res Cell Motil 1990, 11:216-26.

36. Miller JB, Crow MT, Stockdale FE: Slow and fast myosin heavy chain content defines three types of myotubes in early muscle cell cultures. J Cell Biol 1985, 101:1643-50.

doi:10.1186/1471-213X-10-75

Cite this article as: Geach and Zimmerman: Paralysis and delayed Z-disc formation in the Xenopus tropicalis unc45b mutant dicky ticker. BMC Developmental Biology 2010 10:75.

\section{Submit your next manuscript to BioMed Central} and take full advantage of:

- Convenient online submission

- Thorough peer review

- No space constraints or color figure charges

- Immediate publication on acceptance

- Inclusion in PubMed, CAS, Scopus and Google Scholar

- Research which is freely available for redistribution

Submit your manuscript at www.biomedcentral.com/submit
Biomed Central 